\title{
离子液体中吡喃并[3,2-c]吡啶衍生物的绿色合成
}

\author{
张梅梅 $^{a}$ 周玉静 ${ }^{b}$ 李玉玲 ${ }^{b} \quad$ 刘 蕴 ${ }^{b}$ 王香善 $*, a, b$ \\ ( ${ }^{a}$ 江苏师范大学江苏省药用植物生物技术重点实验室 徐州 221116) \\ ( ${ }^{b}$ 江苏师范大学化学化工学院＼cjkstart徐州 221116)
}

\begin{abstract}
摘要 介绍了一种以离子液体为介质合成 2 -氨基-4-芳基-7,8-二氢化-5-氧代- $4 H$-吡喃并 $[3,2-c]$ 吡啶-6-羒酸叔丁酯衍生物 的方法. 该方法包含芳醛、丙二腈或氨乙酸酯和 2,4-二氧代吡啶-1-羧酸叔丁酯的三组分反应，具有产率高和环境友好 的优点.

关键词＼cjkstart吡喃并吡啶; 丙二腈; 离子液体; 合成
\end{abstract}

\section{Green Synthesis of Pyrano[3,2-c]pyridine Derivatives in Ionic Liquids}

\author{
Zhang, Meimei $^{a} \quad$ Zhou, Yujing $^{b} \quad$ Li, Yuling $^{b} \quad$ Liu, Yun $^{b} \quad$ Wang, Xiangshan ${ }^{*, a, b}$ \\ ( ${ }^{a}$ Key Laboratory of Biotechnology on Medical Plant of Jiangsu Province, Jiangsu Normal University, Xuzhou 221116) \\ ( ${ }^{b}$ School of Chemistry and Chemical Engineering, Jiangsu Normal University, Xuzhou 221116)
}

\begin{abstract}
A green and facile method for the synthesis of tert-butyl 2-amino-4-aryl-7,8-dihydro-5-oxo-4H-pyrano$[3,2-c]$ pyridine- $6(5 H)$-carboxylate derivatives, is described in good to high yields using ionic liquids as green media. The method involves a three-component reaction of aromatic aldehyde, malononitrile or cyanacetate and tert-butyl 2,4-dioxopiperidine-1-carboxylate.

Keywords pyranopyridine; malononitrile; ionic liquid; synthesis
\end{abstract}

吡喃并吡啶衍生物是一类重要的化合物，具有诸多 生物和药理活性, 例如抗分支杆菌活性 ${ }^{[1]}$ 、抗血管舒张 活性 ${ }^{[2]}$ 、亲神经活性 ${ }^{[3]}$ 和抗血管新生活性 ${ }^{[4]}$. 另外还被用 作缺氧诱导因子(HIF)抑制剂 ${ }^{[5]}$ 及钾通道拮抗剂 ${ }^{[6]}$. 尽管 已有很多文献报道过其合成方法, 主要包括磺酸催化下 丙二腈和 3,5-二芳亚甲基- $N$-甲基吡啶-4-酮的反应 ${ }^{[7]}$, $\mathrm{Zr}(\mathrm{IV})$ 络合物催化间苯二酚、丙二腈、芳醛和环己酮的 反应 ${ }^{[8]}, 3$-氧代-3-苯基- $N$-(3-吡啶基)丙酰胺和烯胺腈的 反应 ${ }^{[9]}$, 以氨基酸和不饱和醇为原料的 $[4+2]$ 环加成反 应 $^{[10]}$, 及三乙胺催化下醛、吡啶酮和丙二腈的溶剂反应 [11]等多种方法 ${ }^{[12]}$. 但这些方法具有或属于多步反应、或 原料不易得、或需要昂贵的催化剂参与、或使用有机溶 剂作为反应介质等不足.

近年来, 离子液体作为绿色化学的研究内容之一越 来越受到人们的重视. 因为离子液体本身既可以作为溶 剂, 又可以作为反应催化剂, 还具有不易燃, 不易爆,
挥发性小和可以循环使用等优点，在有机合成中，作为 绿色反应介质在很多经典的反应中得到了广泛的应用, 主要包括还原反应 ${ }^{[13]}$ 、氧化反应 ${ }^{[14]}$ 、Friedel-Crafts 反 应 $^{[15]} 、$ Suzuki 偶联 ${ }^{[16]}$ 、Heck 反应 ${ }^{[17]}$ 和 Michael 加成反 应等 ${ }^{[18]}$. 在杂环合成中也已经得到很好的应用 ${ }^{[19]}$. 我们 课题组 ${ }^{[20]}$ 已经成功将离子液体用作介质绿色合成了多 种杂环化合物，本文报道离子液体中 2-氨基-4-芳基-7,8二氢化-5-氧代- $4 H$-吡喃并 $[3,2-c]$ 吡啶-6-羊夋酸叔丁酯衍 生物的合成.

\section{1 结果与讨论}

离子液体 $[\mathrm{BMIm}] \mathrm{Br}$ 中, 将芳醛 1、丙二腈或氰乙酸 酯 2 和 2,4-二氧代吡啶-1-羧酸叔丁酯(3)的三组分 $80{ }^{\circ} \mathrm{C}$ 反应几小时，可以较高产率得到相应的 2-氨基-4-芳基7,8-二氢化-5-氧代- $4 H$-吡喃并 $[3,2-c]$ 吡啶-6-羧酸叔丁酯 衍生物(Eq. 1).

\footnotetext{
*E-mail: xswang1974@yahoo.com

Received February 26, 2013; revised April 9, 2013; published online April 17, 2013.

Project supported by the National Natural Science Foundation of China (Nos. 20802061, 21104064), the Qing Lan Project (Nos. 10QLD008, GSFM2011003) and the College Industrialization Project (No. JHB2012-31) of Jiangsu Province.

国家自然科学基金(Nos. 20802061, 21104064)、青蓝工程基金(Nos. 10QLD008, GSFM2011003)、江苏省高校产业化基金(No. JHB2012-31)资助项目.
} 


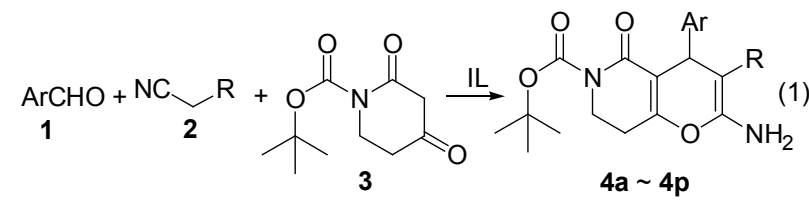

我们用 4-甲基苯甲醛(1a)、丙二腈(2a)和 2,4-二氧代 吡啶-1-羧酸叔丁酯(3)的三组分反应为例, 对反应条件 进行优化篎选(表 1). 在室温下反应通过 TLC 检测并没 有发现产物 $\mathbf{4 a}$; 说明该反应对温度的依赖性很强, 80 ${ }^{\circ} \mathrm{C}$ 下得到最高的产率 $(86 \%)$. 以不同的离子液体, 包括 不同的咪唑阳离子和阴离子作反应介质, 发现 $[\mathrm{BMIm}] \mathrm{Br}$ 对于这个反应的效果最好. 反应结束后, 向 反应体系内加入少量的水析出产物, 简单的过滤即可实 现产物和离子液体分离. 减压除去滤液中的水, $80{ }^{\circ} \mathrm{C}$ 真 空干燥 $4 \mathrm{~h}$, 即可循环使用离子液体, 上述的反应循环 使用离子液体 4 次, 产物 $4 \mathrm{a}$ 的产率还很高( $85 \%$ ).

表 1 不同条件下 $4 \mathrm{a}$ 的合成结果 ${ }^{a}$

Table 1 Synthetic results of $\mathbf{4 a}$ under different reaction conditions

\begin{tabular}{cccc}
\hline 序号 & 温度 $/{ }^{\circ} \mathrm{C}$ & 离子液体 $^{b}$ & 产率 ${ }^{c} / \%$ \\
\hline 1 & r.t. & {$[\mathrm{BMIm}] \mathrm{Br}$} & 0 \\
2 & 50 & {$[\mathrm{BMIm}] \mathrm{Br}$} & 64 \\
3 & 80 & {$[\mathrm{BMIm}] \mathrm{Br}$} & 86 \\
4 & 100 & {$[\mathrm{BMIm}] \mathrm{Br}$} & 86 \\
5 & 80 & {$[\mathrm{EMIm}] \mathrm{Br}$} & 82 \\
6 & 80 & {$[\mathrm{PMIm}] \mathrm{Br}$} & 85 \\
7 & 80 & {$[\mathrm{EMIm}]\left[\mathrm{BF}_{4}\right]$} & 54 \\
8 & 80 & {$\left[\mathrm{PMIm}_{4}\right]\left[\mathrm{BF}_{4}\right]$} & 60 \\
9 & 80 & $\left.[\mathrm{BMIm}] \mathrm{BF}_{4}\right]$ & 58 \\
\hline
\end{tabular}

$a_{\text {反应条件: } 2 \mathrm{~mL} \text { 溶剂, 1a }(0.120 \mathrm{~g}, 1.0 \mathrm{mmol}), \mathbf{2 a}(0.069 \mathrm{~g}, 1.05 \mathrm{mmol}) \text { 和 } \mathbf{3}}$ $(0.213 \mathrm{~g}, 1.0 \mathrm{mmol}){ }^{b} \mathrm{BMIm}$ 为 1 -丁基-3-甲基咪唑阳离子; $\mathrm{EMIm}=1$-乙基3-甲基咪唑阳离子; PMIm $=1$-丙基-3-甲基咪唑阳离子. ${ }^{c}$ 分离产率.

根据篮选和优化的反应条件, 不同的芳醛被选用与 丙二腈和 3 反应, 均得到相应的吡喃并吡啶产物(表 2 , 系列 $1 \sim 10$ ). 用氧乙酸酯替代丙二腈进行该反应, 也得 到很好的结果(表 2, 系列 11 16). 所采用的芳醛, 无论 是邻位、间位还是对位取代; 无论含有吸电子基团(例如 卤素或硝基), 还是供电子基团(例如烷基或烷氧基), 都 没有很大的影响, 均可以得到很好的产率. 通过 IR, ${ }^{1} \mathrm{H}$ $\mathrm{NMR},{ }^{13} \mathrm{C}$ NMR 和 HRMS 表征所有产物的结构, 数据均 与产物 $\mathbf{4}$ 的结构相吻合; 产物 $\mathbf{4 j}$ 的结构还被 X 光衍射 分析确证(图 1, CCDC933303).

在晶体结构 $\mathbf{4 j}$ 中, 吡喃环采用半椅式构象: 原子 $\mathrm{C}(1), \mathrm{C}(2), \mathrm{O}(4), \mathrm{C}(4)$ 和 $\mathrm{C}(8)$ 共平面, 原子 $\mathrm{C}(3)$ 偏离这个 平面为 0.154(3) $\AA$; 这个平面几乎垂直于相连接的苯环, 形成的二面角为 $85.7(1)^{\circ}$. 相邻的吡啶环同样扭曲却采 用扭船式构象: 原子 $\mathrm{C}(4), \mathrm{C}(5), \mathrm{C}(7)$ 和 $\mathrm{C}(8)$ 在一个平面 上, 原子 $\mathrm{C}(6)$ 和 $\mathrm{N}(3)$ 偏离该平面的距离分别为 $0.838(4)$
表 2 离子液体中 $\mathbf{4 a} \sim \mathbf{4 p}$ 的合成结果 ${ }^{a}$

Table 2 Synthetic results of $\mathbf{4 a} \sim \mathbf{4 p}$ in ionic liquids

\begin{tabular}{|c|c|c|c|c|c|}
\hline 序号 & $\mathrm{Ar}$ & $\mathrm{R}$ & $\begin{array}{c}\text { 反应时 } \\
\text { 间 } / \mathrm{h}\end{array}$ & 产物 & $\begin{array}{c}\text { 产率 } \\
\\
1 \% \\
\end{array}$ \\
\hline 1 & $4-\mathrm{MeC}_{6} \mathrm{H}_{4}$ & $\mathrm{CN}$ & 8 & $4 a$ & 86 \\
\hline 2 & $4-\mathrm{FC}_{6} \mathrm{H}_{4}$ & $\mathrm{CN}$ & 5 & $4 b$ & 90 \\
\hline 3 & $3,4-\mathrm{Cl}_{2} \mathrm{C}_{6} \mathrm{H}_{3}$ & $\mathrm{CN}$ & 5 & $4 c$ & 84 \\
\hline 4 & $2-\mathrm{NO}_{2}-3,4-(\mathrm{OMe})_{2} \mathrm{C}_{6} \mathrm{H}_{2}$ & $\mathrm{CN}$ & 3 & $4 d$ & 89 \\
\hline 5 & Furan-2-yl & $\mathrm{CN}$ & 7 & $4 e$ & 82 \\
\hline 6 & $4-\mathrm{ClC}_{6} \mathrm{H}_{4}$ & $\mathrm{CN}$ & 8 & $4 f$ & 88 \\
\hline 7 & $3,4-(\mathrm{OMe})_{2} \mathrm{C}_{6} \mathrm{H}_{3}$ & $\mathrm{CN}$ & 8 & $4 g$ & 78 \\
\hline 8 & $\mathrm{Ph}$ & $\mathrm{CN}$ & 8 & $4 h$ & 89 \\
\hline 9 & $4-\mathrm{NCC}_{6} \mathrm{H}_{4}$ & $\mathrm{CN}$ & 6 & $4 \mathbf{i}$ & 85 \\
\hline 10 & $2-\mathrm{FC}_{6} \mathrm{H}_{4}$ & $\mathrm{CN}$ & 6 & $4 \mathbf{j}$ & 89 \\
\hline 11 & $3-\mathrm{NO}_{2} \mathrm{C}_{6} \mathrm{H}_{4}$ & $\mathrm{CO}_{2} \mathrm{Et}$ & 10 & $4 k$ & 78 \\
\hline 12 & $2-\mathrm{ClC}_{6} \mathrm{H}_{4}$ & $\mathrm{CO}_{2} \mathrm{Et}$ & 12 & 41 & 85 \\
\hline 13 & $4-\mathrm{ClC}_{6} \mathrm{H}_{4}$ & $\mathrm{CO}_{2} \mathrm{Et}$ & 12 & $4 m$ & 79 \\
\hline 14 & $3,4-\mathrm{Cl}_{2} \mathrm{C}_{6} \mathrm{H}_{3}$ & $\mathrm{CO}_{2} \mathrm{Et}$ & 10 & $4 n$ & 80 \\
\hline 15 & $4-\mathrm{MeOC}_{6} \mathrm{H}_{4}$ & $\mathrm{CO}_{2} \mathrm{Et}$ & 12 & 40 & 86 \\
\hline 16 & $2-\mathrm{ClC}_{6} \mathrm{H}_{4}$ & $\mathrm{CO}_{2} \mathrm{Me}$ & 10 & $4 p$ & 76 \\
\hline
\end{tabular}
$\mathrm{mmol}){ }^{b}$ 分离产率.

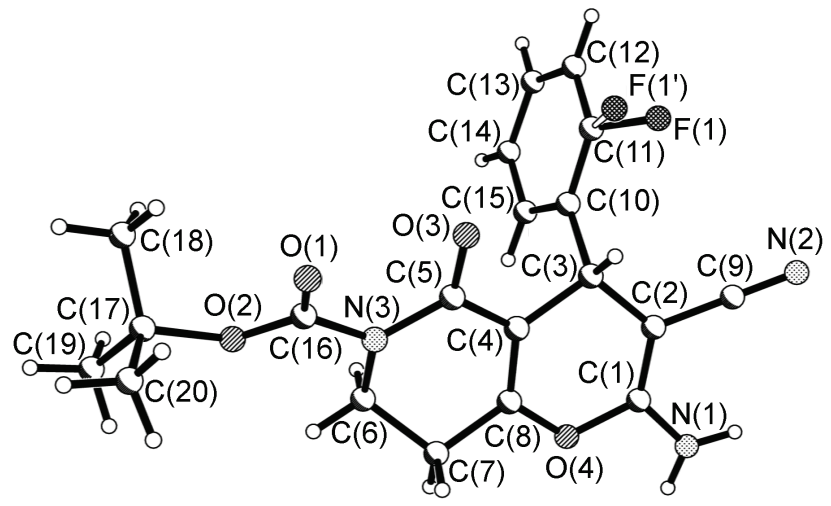

图 1 产物 $4 \mathrm{j}$ 的晶体结构图

Figure 1 The crystal structure of product $\mathbf{4 j}$

和 0.289(4) $\AA$. 两个杂环几乎相互平行, 形成的二面角 为 $8.6(1)^{\circ}$.

\section{2 结论}

本文介绍了三组分一锅法合成吡喃并吡啶衍生物 的方法，具有产率高、反应条件温和、产率高、分离简 单和环境友好等优点. 并通过 IR, ${ }^{1} \mathrm{H} N \mathrm{NMR},{ }^{13} \mathrm{C} \mathrm{NMR}$ 和 HRMS 表征其结构, 其中化合物 $\mathbf{4 j}$ 进一步通过 $X$ 光衍 射分析确证.

\section{3 实验部分}

\section{1 仪器与试剂}

熔点测定使用 XT-5 型数字熔点仪; 红外光谱采用 TENSOR 27 型红外光谱仪测定 $(\mathrm{KBr}$ 压片); 核磁共振氢 
谱采用 Bruker AVANCE DPX-400 型核磁共振仪测定, DMSO- $d_{6}$ 为溶剂, TMS 为内标; 高分辨质谱在 Brukermicro-TOF-Q-MS 质谱仪上测定.

\section{2 实验方法}

干燥的 $50 \mathrm{~mL}$ 烧瓶中加入芳醛(1) $(1.0 \mathrm{mmol})$, 丙 二腈或氰乙酸酯 $(2)(1.05 \mathrm{mmol}), 2,4-$ 二氧代吡啶-1-羧酸 叔丁酯(3) $(0.213 \mathrm{~g}, 1 \mathrm{mmol})$ 和离子液体 $[\mathrm{BMIm}] \mathrm{Br}$ (2 $\mathrm{mL}$ ). 搅拌加热到 $80{ }^{\circ} \mathrm{C}$ 保持 $3 \sim 12 \mathrm{~h}$, 冷却到室温加入 $5 \mathrm{~mL}$ 水, 过滤得到粗品. 粗品用 $95 \% \mathrm{EtOH}$ 重结晶得到 化合物 4.

2-氨基-3-氰基-5-氧代-4-对甲苯基-7,8-二氢化- $4 H$ 吡喃并 $[3,2-c]$ 吡啶- $6(5 H)$-羒酸叔丁酯(4a): m.p. 212 $214{ }^{\circ} \mathrm{C} ;{ }^{1} \mathrm{H}$ NMR $\left(\mathrm{CDCl}_{3}, 400 \mathrm{MHz}\right) \delta: 1.40(\mathrm{~s}, 9 \mathrm{H}$, $\left.3 \mathrm{CH}_{3}\right), 2.30$ (s, $\left.3 \mathrm{H}, \mathrm{CH}_{3}\right), 2.46 \sim 2.53(\mathrm{~m}, 1 \mathrm{H}, \mathrm{CH}), 2.75 \sim$ $2.84(\mathrm{~m}, 1 \mathrm{H}, \mathrm{CH}), 3.48 \sim 3.56(\mathrm{~m}, 1 \mathrm{H}, \mathrm{CH}), 4.20 \sim 4.26$ $(\mathrm{m}, 1 \mathrm{H}, \mathrm{CH}), 4.53 \sim 4.55\left(\mathrm{~m}, 3 \mathrm{H}, \mathrm{CH}+\mathrm{NH}_{2}\right), 7.11(\mathrm{~d}$, $J=8.0 \mathrm{~Hz}, 2 \mathrm{H}, \operatorname{ArH}), 7.17(\mathrm{~d}, J=8.0 \mathrm{~Hz}, 2 \mathrm{H}, \operatorname{ArH}) ;{ }^{13} \mathrm{C}$ NMR (DMSO- $\left.d_{6}, 100 \mathrm{MHz}\right) \delta: 163.1,159.3,158.6,158.5$, $152.3,142.1,136.3,129.4,127.7,120.2,108.5,82.4,58.7$, 36.4, 28.1, 26.1, 21.1; IR (KBr) v: 3377, 3306, 3211, 3168, 3050, 2980, 2925, 2194, 1764, 1696, 1676, 1644, 1590, 1514, 1470, 1371, 1329, 1307, 1223, 1164, 1144, 1083, 1044, 1009, 951, 851, 793, 772, $755 \mathrm{~cm}^{-1}$; HRMS calcd for $\mathrm{C}_{21} \mathrm{H}_{23} \mathrm{~N}_{3} \mathrm{O}_{4} \mathrm{Na}[\mathrm{M}+\mathrm{Na}]^{+}$404.1586, found 404.1602.

2-氨基-3-氧基-5-氧代-4-对氟苯基-7,8-二氢化- $4 \mathrm{H}$ 吡喃并 $[3,2-c]$ 吡啶- $6(5 H)$-羧酸叔丁酯(4b): m.p. 185 $187{ }^{\circ} \mathrm{C} ;{ }^{1} \mathrm{H}$ NMR (DMSO- $\left.d_{6}, 400 \mathrm{MHz}\right) \delta: 1.41$ (s, 9H, $\left.3 \mathrm{CH}_{3}\right), 2.57 \sim 2.63(\mathrm{~m}, 1 \mathrm{H}, \mathrm{CH}), 2.80 \sim 2.87(\mathrm{~m}, 1 \mathrm{H}, \mathrm{CH})$, $3.54 \sim 3.61(\mathrm{~m}, 1 \mathrm{H}, \mathrm{CH}), 3.98 \sim 4.03(\mathrm{~m}, 1 \mathrm{H}, \mathrm{CH}), 4.29(\mathrm{~s}$, $1 \mathrm{H}, \mathrm{CH}), 7.06$ (s, 2H, NH$\left.)_{2}\right), 7.12$ (t, $J=8.8 \mathrm{~Hz}, 2 \mathrm{H}, \mathrm{ArH}$ ), $7.23 \sim 7.29(\mathrm{~m}, 2 \mathrm{H}, \mathrm{ArH}) ;{ }^{13} \mathrm{C}$ NMR (DMSO- $d_{6}, 100$ MHz) $\delta$ : $163.1,161.5\left(\mathrm{~d}, J_{\mathrm{C}-\mathrm{F}}=241.0 \mathrm{~Hz}\right), 159.6,158.60$, 158.56, 152.3, $141.2\left(\mathrm{~d}, J_{\mathrm{C}-\mathrm{F}}=2.7 \mathrm{~Hz}\right), 129.7\left(\mathrm{~d}, J_{\mathrm{C}-\mathrm{F}}=\right.$ $8.1 \mathrm{~Hz}), 120.0,115.5\left(\mathrm{~d}, J_{\mathrm{C}-\mathrm{F}}=21.2 \mathrm{~Hz}\right), 108.2,82.6$, 58.4, 36.1, 28.0, 26.1; IR (KBr) v: 3383, 3305, 3211, 3171, 3064, 2981, 2930, 2193, 1765, 1694, 1676, 1644, 1591, 1508, 1473, 1371, 1307, 1222, 1161, 1144, 1094, 1083, 1044, 1011, 952, 852, 802, 777, $760 \mathrm{~cm}^{-1}$; HRMS calcd for $\mathrm{C}_{20} \mathrm{H}_{20} \mathrm{~N}_{3} \mathrm{O}_{4} \mathrm{FNa}[\mathrm{M}+\mathrm{Na}]^{+} 408.1336$, found 408.1335 .

2-氨基-3-氰基-5-氧代-4-(3,4-二氯苯基)-7,8-二氢 化-4H-吡喃并 [3,2-c]吡啶-6(5H)-羧酸叔丁酯 (4c): m.p. $205 \sim 207{ }^{\circ} \mathrm{C} ;{ }^{1} \mathrm{H}$ NMR (DMSO- $\left.d_{6}, 400 \mathrm{MHz}\right) \delta: 1.40$ (s, $\left.9 \mathrm{H}, 3 \mathrm{CH}_{3}\right), 2.59 \sim 2.64(\mathrm{~m}, 1 \mathrm{H}, \mathrm{CH}), 2.78 \sim 2.86(\mathrm{~m}, 1 \mathrm{H}$, $\mathrm{CH}), 3.58 \sim 3.64(\mathrm{~m}, 1 \mathrm{H}, \mathrm{CH}), 3.97 \sim 4.00(\mathrm{~m}, 1 \mathrm{H}, \mathrm{CH})$, $4.32(\mathrm{~s}, 1 \mathrm{H}, \mathrm{CH}), 7.17\left(\mathrm{~s}, 2 \mathrm{H}, \mathrm{NH}_{2}\right), 7.23(\mathrm{~d}, J=8.0 \mathrm{~Hz}$,
1H, ArH), 7.47 (s, 1H, ArH), $7.56(\mathrm{~d}, J=8.0 \mathrm{~Hz}, 1 \mathrm{H}$, ArH); ${ }^{13} \mathrm{C}$ NMR (DMSO- $\left.d_{6}, 100 \mathrm{MHz}\right) \delta: 163.1,160.2$, $158.7,158.6,152.2,146.2,131.4,131.1,129.9,129.8$, $128.4,119.8,107.3,82.5,57.6,36.2,28.1,26.2$; IR (KBr) $v: 3391,3319,3272,3229,3198,2976,2931,2189,1722$, 1699, 1666, 1645, 1607, 1471, 1405, 1369, 1307, 1228, 1150, 1084, 1050, 1029, 953, 878, 848, 778, 755, 725 $\mathrm{cm}^{-1}$; HRMS calcd for $\mathrm{C}_{20} \mathrm{H}_{19} \mathrm{~N}_{3} \mathrm{O}_{4} \mathrm{Cl}_{2} \mathrm{Na}[\mathrm{M}+\mathrm{Na}]^{+}$ 458.0650 , found 458.0643 .

2-氨基-3-氧基-5-氧代-4-(2-硝基-3,4-二甲氧基苯 基)-7,8-二氢化- $4 H$-吡喃并 $[3,2-c]$ 吡啶- $6(5 H)$-羧酸叔丁 酯(4d): m.p. $184 \sim 186{ }^{\circ} \mathrm{C} ;{ }^{1} \mathrm{H}$ NMR (DMSO- $d_{6}, 400$ $\mathrm{MHz}) \delta: 1.39\left(\mathrm{~s}, 9 \mathrm{H}, 3 \mathrm{CH}_{3}\right), 2.60 \sim 2.64(\mathrm{~m}, 1 \mathrm{H}, \mathrm{CH})$, $2.77 \sim 2.86(\mathrm{~m}, 1 \mathrm{H}, \mathrm{CH}), 3.57 \sim 3.65(\mathrm{~m}, 1 \mathrm{H}, \mathrm{CH}), 3.84(\mathrm{~s}$, $\left.6 \mathrm{H}, 2 \mathrm{OCH}_{3}\right), 3.97 \sim 4.01(\mathrm{~m}, 1 \mathrm{H}, \mathrm{CH}), 5.19(\mathrm{~s}, 1 \mathrm{H}, \mathrm{CH})$, 6.75 (s, 1H, ArH), 7.13 (s, 2H, NH $\mathrm{NH}_{2}, 7.48$ (s, 1H, ArH); ${ }^{13} \mathrm{C}$ NMR (DMSO- $\left.d_{6}, 100 \mathrm{MHz}\right) \delta: 163.1,160.0,159.13$, $159.09,153.3,151.9,147.7,141.7,133.6,119.5,111.8$, $107.9,107.7,82.5,56.8,56.6,56.5,36.3,28.1,26.1$; IR (KBr) $v: 3320,3195,2979,2937,2849,2188,1759,1694$, $1677,1639,1610,1517,1448,1393,1370,1329,1307$, 1272, 1224, 1140, 1089, 1052, 954, 870, 847, 799, 778, $748,717 \mathrm{~cm}^{-1}$; HRMS calcd for $\mathrm{C}_{22} \mathrm{H}_{24} \mathrm{~N}_{4} \mathrm{O}_{8} \mathrm{Na}[\mathrm{M}+\mathrm{Na}]^{+}$ 495.1492, found 495.1468.

2-氨基-3-氧基-5-氧代-4-(2-呋喃基)-7,8-二氢化-4H吡喃并 $[3,2-c]$ 吡啶-6(5H)-羧酸叔丁酯(4e): m.p. 198 $200{ }^{\circ} \mathrm{C} ;{ }^{1} \mathrm{H}$ NMR (DMSO- $\left.d_{6}, 400 \mathrm{MHz}\right) \delta: 1.43$ (s, 9H, $\left.3 \mathrm{CH}_{3}\right), 2.57 \sim 2.62(\mathrm{~m}, 1 \mathrm{H}, \mathrm{CH}), 2.78 \sim 2.85(\mathrm{~m}, 1 \mathrm{H}, \mathrm{CH})$, $3.55 \sim 3.61(\mathrm{~m}, 1 \mathrm{H}, \mathrm{CH}), 4.00 \sim 4.04(\mathrm{~m}, 1 \mathrm{H}, \mathrm{CH}), 4.11(\mathrm{~s}$, $1 \mathrm{H}, \mathrm{CH}), 6.14$ (s, 1H, ArH), 6.35 (s, 1H, ArH), $7.12(\mathrm{~s}, 2 \mathrm{H}$, $\mathrm{NH}_{2}$ ), $7.52(\mathrm{~s}, 1 \mathrm{H}, \mathrm{ArH}) ;{ }^{13} \mathrm{C}$ NMR (DMSO- $d_{6}, 100 \mathrm{MHz}$ ) $\delta: 162.9,160.2,159.50,159.46,156.0,152.3,142.5,120.0$, $111.0,106.00,105.95,82.5,55.7,30.4,28.1,26.1$; IR (KBr) $v: 3381,3315,3200,2987,2935,2891,2196,1764$, 1698, 1647, 1613, 1504, 1471, 1395, 1373, 1334, 1304, $1222,1137,1085,1052,1007,950,849,804,768,740$ $\mathrm{cm}^{-1}$; HRMS calcd for $\mathrm{C}_{18} \mathrm{H}_{19} \mathrm{~N}_{3} \mathrm{O}_{5} \mathrm{Na}[\mathrm{M}+\mathrm{Na}]^{+}$ 380.1222 , found 380.1203 .

2-氨基-3-氭基-5-氧代-4-对氯苯基-7,8-二氢化-4H吡喃并[3,2-c]吡啶-6(5H)-羧酸叔丁酯(4f): m.p. 193 195 ${ }^{\circ} \mathrm{C} ;{ }^{1} \mathrm{H}$ NMR (DMSO- $\left.d_{6}, 400 \mathrm{MHz}\right) \delta: 1.41\left(\mathrm{~s}, 9 \mathrm{H}, 3 \mathrm{CH}_{3}\right.$ ), $2.57 \sim 2.63(\mathrm{~m}, 1 \mathrm{H}, \mathrm{CH}), 2.79 \sim 2.88(\mathrm{~m}, 1 \mathrm{H}, \mathrm{CH}), 3.54 \sim$ $3.62(\mathrm{~m}, 1 \mathrm{H}, \mathrm{CH}), 3.98 \sim 4.03(\mathrm{~m}, 1 \mathrm{H}, \mathrm{CH}), 4.28(\mathrm{~s}, 1 \mathrm{H}$, $\mathrm{CH}), 7.08\left(\mathrm{~s}, 2 \mathrm{H}, \mathrm{NH}_{2}\right), 7.24$ (d, $\left.J=8.4 \mathrm{~Hz}, 2 \mathrm{H}, \mathrm{ArH}\right), 7.37$ $(\mathrm{d}, J=8.4 \mathrm{~Hz}, 2 \mathrm{H}, \mathrm{ArH}) ;{ }^{13} \mathrm{C}$ NMR (DMSO- $d_{6}, 100 \mathrm{MHz}$ ) 
$\delta: 163.1,159.8,158.63,158.59,152.3,144.0,131.7,129.8$, 128.8, 120.0, 107.9, 82.5, 58.1, 36.3, 28.1, 26.1; IR (KBr) v: 3444, 3317, 3274, 3225, 3193, 2979, 2936, 2892, 2201, 1764, 1698, 1660, 1645, 1605, 1489, 1394, 1370, 1299, 1218, 1160, 1141, 1091, 1054, 1015, 954, 836, $765 \mathrm{~cm}^{-1}$; HRMS calcd for $\mathrm{C}_{20} \mathrm{H}_{19} \mathrm{~N}_{3} \mathrm{O}_{4} \mathrm{Cl}[\mathrm{M}-\mathrm{H}]^{-}$400.1064, found 400.1074 .

2-氨基-3-氧基-5-氧代-4-(3,4-二甲氧基苯基)-7,8-二 氢化-4H-吡喃并 $[3,2-c]$ 吡啶- $6(5 H)$-羧酸叔丁酯 $(4 \mathrm{~g})$ : m.p. 197 $199{ }^{\circ} \mathrm{C} ;{ }^{1} \mathrm{H}$ NMR (DMSO- $\left.d_{6}, 400 \mathrm{MHz}\right) \delta: 1.41$ (s, $\left.9 \mathrm{H}, 3 \mathrm{CH}_{3}\right), 2.57 \sim 2.63(\mathrm{~m}, 1 \mathrm{H}, \mathrm{CH}), 2.80 \sim 2.87(\mathrm{~m}, 1 \mathrm{H}$, $\mathrm{CH}), 3.53 \sim 3.60(\mathrm{~m}, 1 \mathrm{H}, \mathrm{CH}), 3.72\left(\mathrm{~s}, 3 \mathrm{H}, \mathrm{OCH}_{3}\right), 3.74(\mathrm{~s}$, $\left.3 \mathrm{H}, \mathrm{OCH}_{3}\right), 4.00 \sim 4.05(\mathrm{~m}, 1 \mathrm{H}, \mathrm{CH}), 4.22(\mathrm{~s}, 1 \mathrm{H}, \mathrm{CH})$, $6.70 \sim 6.72(\mathrm{~m}, 1 \mathrm{H}, \mathrm{ArH}), 6.75 \sim 6.76(\mathrm{~m}, 1 \mathrm{H}, \mathrm{ArH}), 6.88$ (d, $J=8.4 \mathrm{~Hz}, 1 \mathrm{H}, \mathrm{ArH}), 6.97$ (s, $\left.2 \mathrm{H}, \mathrm{NH}_{2}\right) ;{ }^{13} \mathrm{C} \mathrm{NMR}$ (DMSO- $\left.d_{6}, 100 \mathrm{MHz}\right) \delta: 163.1,159.3,158.50,158.46$, $152.3,149.0,148.1,137.5,120.2,119.7,112.3,111.7$, $108.4,82.4,58.8,56.5,56.0,36.2,28.1,26.2$; IR (KBr) $v$ : $3416,3320,3190,2972,2937,2883,2836,2201,1754$, $1691,1665,1632,1611,1511,1463,1420,1375,1312$, 1271, 1228, 1144, 1051, 1028, 949, 851, 803, 776, 762, $735 \mathrm{~cm}^{-1}$; HRMS calcd for $\mathrm{C}_{22} \mathrm{H}_{25} \mathrm{~N}_{3} \mathrm{O}_{6} \mathrm{Na}[\mathrm{M}+\mathrm{Na}]^{+}$ 450.1641 , found 450.1638 .

2-氨基-3-氧基-5-氧代-4-苯基-7,8-二氢化- $4 H$-吡喃 并[3,2-c]吡啶-6(5H)-羧酸叔丁酯(4h): m.p. $230 \sim 232{ }^{\circ} \mathrm{C}$; ${ }^{1} \mathrm{H}$ NMR (DMSO- $\left.d_{6}, 400 \mathrm{MHz}\right) \delta: 1.41\left(\mathrm{~s}, 9 \mathrm{H}, 3 \mathrm{CH}_{3}\right)$, $2.58 \sim 2.64(\mathrm{~m}, 1 \mathrm{H}, \mathrm{CH}), 2.80 \sim 2.88(\mathrm{~m}, 1 \mathrm{H}, \mathrm{CH}), 3.53 \sim$ $3.61(\mathrm{~m}, 1 \mathrm{H}, \mathrm{CH}), 3.99 \sim 4.05(\mathrm{~m}, 1 \mathrm{H}, \mathrm{CH}), 4.26(\mathrm{~s}, 1 \mathrm{H}$, $\mathrm{CH}), 7.02\left(\mathrm{~s}, 2 \mathrm{H}, \mathrm{NH}_{2}\right), 7.20 \sim 7.23(\mathrm{~m}, 3 \mathrm{H}, \mathrm{ArH}), 7.30 \sim$ 7.33 (m, $2 \mathrm{H}, \mathrm{ArH}) ;{ }^{13} \mathrm{C}$ NMR (DMSO- $\left.d_{6}, 100 \mathrm{MHz}\right) \delta$ : 163.1, 159.6, 158.62, 158.58, 152.3, 145.0, 128.8, 127.8, 127.2, 120.1, 108.3, 82.4, 58.6, 36.8, 28.1, 26.1; IR (KBr) $v: 3460,3322,3269,3221,3192,3024,2978,2196,1759$, $1699,1663,1644,1602,1469,1455,1392,1371,1330$, 1304, 1224, 1161, 1142, 1083, 1056, 952, 850, 837, 785, $737,710,697 \mathrm{~cm}^{-1}$; HRMS calcd for $\mathrm{C}_{20} \mathrm{H}_{21} \mathrm{~N}_{3} \mathrm{O}_{4} \mathrm{Na}[\mathrm{M}+$ $\mathrm{Na}]^{+} 390.1430$, found 390.1433 .

2-氨基-3-氧基-5-氧代-4-对氧基苯基-7,8-二氢化$4 H$-吡喃并 $[3,2-c]$ 吡啶- $6(5 H)$-羧酸叔丁酯(4i): m.p. 190 $192{ }^{\circ} \mathrm{C} ;{ }^{1} \mathrm{H}$ NMR (DMSO- $\left.d_{6}, 400 \mathrm{MHz}\right) \delta: 1.40(\mathrm{~s}, 9 \mathrm{H}$, $\left.3 \mathrm{CH}_{3}\right), 2.60 \sim 2.66(\mathrm{~m}, 1 \mathrm{H}, \mathrm{CH}), 2.80 \sim 2.88(\mathrm{~m}, 1 \mathrm{H}, \mathrm{CH})$, $3.58 \sim 3.66(\mathrm{~m}, 1 \mathrm{H}, \mathrm{CH}), 3.97 \sim 4.02(\mathrm{~m}, 1 \mathrm{H}, \mathrm{CH}), 4.37(\mathrm{~s}$, $1 \mathrm{H}, \mathrm{CH}), 7.16$ (s, $2 \mathrm{H}, \mathrm{NH}_{2}$ ), 7.43 (d, $\left.J=8.4 \mathrm{~Hz}, 2 \mathrm{H}, \mathrm{ArH}\right)$, 7.79 (d, $J=8.4 \mathrm{~Hz}, 2 \mathrm{H}, \mathrm{ArH}) ;{ }^{13} \mathrm{C}$ NMR (DMSO- $d_{6}, 100$ MHz) $\delta: 163.1,160.3,158.70,158.66,152.3,150.5,132.9$,
129.0, 119.8, 119.3, 110.0, 109.3, 82.5, 57.3, 37.0, 28.1, 26.1; IR (KBr) v: 3444, 3317, 3274, 3225, 3193, 2979, 2936, 2892, 2230, 2201, 1764, 1698, 1660, 1645, 1605, 1489, 1394, 1370, 1299, 1218, 1160, 1141, 1091, 1054, $1015,954,836,765 \mathrm{~cm}^{-1}$; HRMS calcd for $\mathrm{C}_{21} \mathrm{H}_{20} \mathrm{~N}_{4} \mathrm{O}_{4} \mathrm{Na}$ $[\mathrm{M}+\mathrm{Na}]^{+} 415.1382$, found 415.1380 .

2-氨基-3-氧基-5-氧代-4-邻氟苯基-7,8-二氢化-4H吡喃并 $[3,2-c]$ 吡啶- $6(5 H)$-羧酸叔丁酯(4j): m.p. 208 210 ${ }^{\circ} \mathrm{C} ;{ }^{1} \mathrm{H}$ NMR (DMSO- $\left.d_{6}, 400 \mathrm{MHz}\right) \delta: 1.40\left(\mathrm{~s}, 9 \mathrm{H}, 3 \mathrm{CH}_{3}\right)$, $2.59 \sim 2.65(\mathrm{~m}, 1 \mathrm{H}, \mathrm{CH}), 2.79 \sim 2.85(\mathrm{~m}, 1 \mathrm{H}, \mathrm{CH}), 3.56 \sim$ $3.63(\mathrm{~m}, 1 \mathrm{H}, \mathrm{CH}), 3.97 \sim 4.03(\mathrm{~m}, 1 \mathrm{H}, \mathrm{CH}), 4.55(\mathrm{~s}, 1 \mathrm{H}$, $\mathrm{CH}), 7.09$ (s, 2H, NH$\left.)_{2}\right), 7.11 \sim 7.16(\mathrm{~m}, 2 \mathrm{H}, \mathrm{ArH}), 7.23 \sim$ 7.27 (m, $2 \mathrm{H}, \mathrm{ArH}) ;{ }^{13} \mathrm{C}$ NMR (DMSO- $\left.d_{6}, 100 \mathrm{MHz}\right) \delta$ : $163.0,160.34\left(\mathrm{~d}, J_{\mathrm{C}-\mathrm{F}}=244.2 \mathrm{~Hz}\right), 160.27,159.94$, $158.89,152.2,131.5\left(\mathrm{~d}, J_{\mathrm{C}-\mathrm{F}}=12.8 \mathrm{~Hz}\right), 130.1\left(\mathrm{~d}, J_{\mathrm{C}-\mathrm{F}}=\right.$ $3.9 \mathrm{~Hz}), 129.2\left(\mathrm{~d}, J_{\mathrm{C}-\mathrm{F}}=8.0 \mathrm{~Hz}\right), 125.1\left(\mathrm{~d}, J_{\mathrm{C}-\mathrm{F}}=3.1 \mathrm{~Hz}\right)$, $119.9,115.9\left(\mathrm{~d}, J_{\mathrm{C}^{-} \mathrm{F}}=21.7 \mathrm{~Hz}\right), 107.0,82.5,57.1,30.7$, 28.1, 26.1; IR (KBr) v: 3467, 3322, 3265, 3218, 3189, 2976, 2932, 2196, 1758, 1700, 1662, 1643, 1601, 1487, 1457, 1392, 1372, 1329, 1306, 1258, 1221, 1162, 1143, 1096, 1058, 954, 845, 806, 778, 758, $705 \mathrm{~cm}^{-1}$; HRMS calcd for $\mathrm{C}_{20} \mathrm{H}_{20} \mathrm{~N}_{3} \mathrm{O}_{4} \mathrm{~F}[\mathrm{M}+\mathrm{Na}]^{+}$408.1336, found 408.1347 .

2-氨基-3-乙氧基羰基-5-氧代-4-间硝基苯基-7,8-二 氢化- $4 H$-吡喃并 $[3,2-c]$ 吡啶- $6(5 H)$-羧酸叔丁酯 $(4 \mathbf{k}):$ m.p. 194 $196{ }^{\circ} \mathrm{C} ;{ }^{1} \mathrm{H}$ NMR (DMSO- $\left.d_{6}, 400 \mathrm{MHz}\right) \delta: 1.07$ (t, $\left.J=6.8 \mathrm{~Hz}, 3 \mathrm{H}, \mathrm{CH}_{3}\right), 1.41\left(\mathrm{~s}, 9 \mathrm{H}, 3 \mathrm{CH}_{3}\right), 2.65 \sim 2.71(\mathrm{~m}$, $1 \mathrm{H}, \mathrm{CH}), 2.81 \sim 2.88(\mathrm{~m}, 1 \mathrm{H}, \mathrm{CH}), 3.52 \sim 3.59(\mathrm{~m}, 1 \mathrm{H}$, $\mathrm{CH}), 3.92 \sim 4.02\left(\mathrm{~m}, 3 \mathrm{H}, \mathrm{CH}_{2}+\mathrm{CH}\right), 4.69(\mathrm{~s}, 1 \mathrm{H}, \mathrm{CH})$, $7.53 \sim 7.57(\mathrm{~m}, 1 \mathrm{H}, \mathrm{ArH}), 7.66 \sim 7.71(\mathrm{~m}, 3 \mathrm{H}, \mathrm{ArH}+$ $\mathrm{NH}_{2}$ ), $8.00 \sim 8.03(\mathrm{~m}, 2 \mathrm{H}, \mathrm{ArH}),{ }^{13} \mathrm{C}$ NMR (DMSO- $d_{6}, 100$ MHz) $\delta: 168.1,163.5,159.70,159.69,159.3,152.3,149.0$, 147.8, 135.3, 129.9, 123.1, 121.7, 110.0, 82.4, 76.9, 59.5, 35.1, 28.1, 26.0, 14.5; IR (KBr) v: 3390, 3283, 2988, 2972, 2933, 2905, 1756, 1700, 1673, 1613, 1530, 1515, 1372, 1350, 1307, 1292, 1229, 1204, 1165, 1143, 1097, 1054, 956, 888, 852, 788, 737, $707 \mathrm{~cm}^{-1}$; HRMS calcd for $\mathrm{C}_{22} \mathrm{H}_{25} \mathrm{~N}_{3} \mathrm{O}_{8} \mathrm{Na}[\mathrm{M}+\mathrm{Na}]^{+} 482.1539$, found 482.1559 .

2-氨基-3-乙氧基羰基-5-氧代-4-邻氯苯基-7,8-二氢 化-4H-吡喃并 $[3,2-c]$ 吡啶-6(5H)-羧酸叔丁酯(4l): m.p. 197 199 ${ }^{\circ} \mathrm{C} ;{ }^{1} \mathrm{H}$ NMR (DMSO- $\left.d_{6}, 400 \mathrm{MHz}\right) \delta$ : 1.03 (t, $\left.J=6.8 \mathrm{~Hz}, 3 \mathrm{H}, \mathrm{CH}_{3}\right), 1.41\left(\mathrm{~s}, 9 \mathrm{H}, 3 \mathrm{CH}_{3}\right), 2.57 \sim 2.63(\mathrm{~m}$, $1 \mathrm{H}, \mathrm{CH}), 2.79 \sim 2.87(\mathrm{~m}, 1 \mathrm{H}, \mathrm{CH}), 3.42 \sim 3.50(\mathrm{~m}, 1 \mathrm{H}$, $\mathrm{CH}), 3.89 \sim 4.00\left(\mathrm{~m}, 3 \mathrm{H}, \mathrm{CH}_{2}+\mathrm{CH}\right), 4.90 \sim 4.91(\mathrm{~s}, 1 \mathrm{H}$, $\mathrm{CH}), 7.12 \sim 7.16(\mathrm{~m}, 1 \mathrm{H}, \mathrm{ArH}), 7.20 \sim 7.24(\mathrm{~m}, 1 \mathrm{H}, \mathrm{ArH})$, 
$7.25 \sim 7.28(\mathrm{~m}, 2 \mathrm{H}, \mathrm{ArH}), 7.64 \sim 7.65\left(\mathrm{~m}, 2 \mathrm{H}, \mathrm{NH}_{2}\right) ;{ }^{13} \mathrm{C}$ NMR (DMSO- $\left.d_{6}, 100 \mathrm{MHz}\right) \delta: 168.5,163.3,159.52$, $159.46,159.3,152.1,142.9,133.2,132.8,129.9,128.1$, 127.0, 109.4, 82.3, 76.5, 59.2, 33.7, 28.1, 26.0, 14.6; IR (KBr) $v$ : 3462, 3308, 2985, 2941, 1751, 1700, 1666, 1609, $1509,1473,1457,1391,1372,1303,1200,1166,1144$, 1073, 1043, 952, 851, 827, 785, 751, $705 \mathrm{~cm}^{-1}$; HRMS calcd for $\mathrm{C}_{22} \mathrm{H}_{25} \mathrm{~N}_{2} \mathrm{O}_{6} \mathrm{ClNa}[\mathrm{M}+\mathrm{Na}]^{+}$471.1299, found 471.1294.

2-氨基-3-乙氧基羰基-5-氧代-4-对氯苯基-7,8-二氢 化-4H-吡喃并 $[3,2-c]$ 吡啶-6(5H)-羧酸叔丁酯 $(4 \mathbf{m})$ : m.p. 194 $196{ }^{\circ} \mathrm{C} ;{ }^{1} \mathrm{H}$ NMR (DMSO- $\left.d_{6}, 400 \mathrm{MHz}\right) \delta: 1.09$ (t, $\left.J=7.2 \mathrm{~Hz}, 3 \mathrm{H}, \mathrm{CH}_{3}\right), 1.41\left(\mathrm{~s}, 9 \mathrm{H}, 3 \mathrm{CH}_{3}\right), 2.59 \sim 2.65(\mathrm{~m}$, $1 \mathrm{H}, \mathrm{CH}), 2.78 \sim 2.87(\mathrm{~m}, 1 \mathrm{H}, \mathrm{CH}), 3.46 \sim 3.53(\mathrm{~m}, 1 \mathrm{H}$, $\mathrm{CH}), 3.93 \sim 4.02\left(\mathrm{~m}, 3 \mathrm{H}, \mathrm{CH}_{2}+\mathrm{CH}\right), 4.57(\mathrm{~s}, 1 \mathrm{H}, \mathrm{CH})$, $7.20(\mathrm{~d}, J=8.4 \mathrm{~Hz}, 2 \mathrm{H}, \mathrm{ArH}), 7.28(\mathrm{~d}, J=8.4 \mathrm{~Hz}, 2 \mathrm{H}$, ArH), 7.60 (s, $2 \mathrm{H}, \mathrm{NH}_{2}$ ); ${ }^{13} \mathrm{C}$ NMR (DMSO- $d_{6}, 100 \mathrm{MHz}$ ) $\delta: 168.3,163.5,159.33,159.27,159.1,152.4,145.8,130.9$, 130.3, 128.2, 110.8, 82.4, 77.4, 59.4, 34.4, 28.1, 26.0, 14.7; IR (KBr) v: 3480, 3340, 2977, 2931, 2904, 1757, 1699, 1674, 1524, 1489, 1468, 1394, 1371, 1315, 1204, 1167, 1146, 1105, 1075, 1051, 1013, 954, 852, 839, $782 \mathrm{~cm}^{-1}$; HRMS calcd for $\mathrm{C}_{22} \mathrm{H}_{25} \mathrm{~N}_{2} \mathrm{O}_{6} \mathrm{ClNa}[\mathrm{M}+\mathrm{Na}]^{+}$471.1299, found 471.1298 .

2-氨基-3-乙氧基羰基-5-氧代-4-(3,4-二氯苯基)-7,8二氢化- $4 H$-吡喃并 $[3,2-c]$ 吡啶- $6(5 H)$-羧酸叔丁酯 $(\mathbf{4 n})$ : m.p. $193 \sim 195{ }^{\circ} \mathrm{C} ;{ }^{1} \mathrm{H}$ NMR (DMSO- $\left.d_{6}, 400 \mathrm{MHz}\right) \delta$ : $1.09\left(\mathrm{t}, J=6.8 \mathrm{~Hz}, 3 \mathrm{H}, \mathrm{CH}_{3}\right), 1.41\left(\mathrm{~s}, 9 \mathrm{H}, 3 \mathrm{CH}_{3}\right), 2.61 \sim$ $2.68(\mathrm{~m}, 1 \mathrm{H}, \mathrm{CH}), 2.79 \sim 2.86(\mathrm{~m}, 1 \mathrm{H}, \mathrm{CH}), 3.50 \sim 3.57$ (m, $1 \mathrm{H}, \mathrm{CH}), 3.91 \sim 4.01\left(\mathrm{~m}, 3 \mathrm{H}, \mathrm{CH}_{2}+\mathrm{CH}\right), 4.54(\mathrm{~s}, 1 \mathrm{H}$, $\mathrm{CH}), 7.17$ (dd, $J=8.4,2.0 \mathrm{~Hz}, 1 \mathrm{H}, \mathrm{ArH}), 7.36 \sim 7.37$ (m, $1 \mathrm{H}, \mathrm{ArH}), 7.54(\mathrm{~d}, J=8.4 \mathrm{~Hz}, 1 \mathrm{H}, \mathrm{ArH}), 7.67 \sim 7.68(\mathrm{~m}$, $2 \mathrm{H}, \mathrm{NH}_{2}$ ) $;{ }^{13} \mathrm{C}$ NMR (DMSO- $\left.d_{6}, 100 \mathrm{MHz}\right) \delta: 168.1$, $163.5,159.5,159.3,159.2,152.3,147.9,130.59,130.58$, $130.5,128.9,128.8,110.0,82.4,76.8,59.4,34.6,28.1$, 26.0. 14.6; IR (KBr) v: 3392, 3285, 2982, 2934, 2907, $1754,1700,1616,1523,1468,1397,1372,1306,1229$, $1203,1143,1074,1047,954,888,849,804,783,729 \mathrm{~cm}^{-}$ '; HRMS calcd for $\mathrm{C}_{22} \mathrm{H}_{24} \mathrm{~N}_{2} \mathrm{O}_{6} \mathrm{Cl}_{2}[\mathrm{M}+\mathrm{Na}]^{+}$505.0909, found 505.0915.

2-氨基-3-乙氧基羰基-5-氧代-4-对甲氧基苯基-7,8二氢化- $4 H$-吡喃并 $[3,2-c]$ 吡啶- $6(5 H)$ - 羧酸叔丁酯 $(\mathbf{4 0})$ : m.p. $198 \sim 199{ }^{\circ} \mathrm{C}$; ${ }^{1} \mathrm{H}$ NMR (DMSO- $\left.d_{6}, 400 \mathrm{MHz}\right) \delta$ : $1.11\left(\mathrm{t}, J=7.2 \mathrm{~Hz}, 3 \mathrm{H}, \mathrm{CH}_{3}\right), 1.42\left(\mathrm{~s}, 9 \mathrm{H}, 3 \mathrm{CH}_{3}\right), 2.58 \sim$ $2.64(\mathrm{~m}, 1 \mathrm{H}, \mathrm{CH}), 2.78 \sim 2.87(\mathrm{~m}, 1 \mathrm{H}, \mathrm{CH}), 3.43 \sim 3.51$ $(\mathrm{m}, 1 \mathrm{H}, \mathrm{CH}), 3.69\left(\mathrm{~s}, 3 \mathrm{H}, \mathrm{OCH}_{3}\right), 3.92 \sim 4.02(\mathrm{~m}, 3 \mathrm{H}$, $\left.\mathrm{CH}_{2}+\mathrm{CH}\right), 4.53(\mathrm{~s}, 1 \mathrm{H}, \mathrm{CH}), 6.78(\mathrm{~d}, J=8.8 \mathrm{~Hz}, 2 \mathrm{H}$, $\mathrm{ArH}), 7.09$ (d, $J=8.8 \mathrm{~Hz}, 2 \mathrm{H}, \mathrm{ArH}), 7.52\left(\mathrm{~s}, 2 \mathrm{H}, \mathrm{NH}_{2}\right) ;{ }^{13} \mathrm{C}$ NMR (DMSO- $\left.d_{6}, 100 \mathrm{MHz}\right) \delta: 168.5,163.5,159.28$, $159.22,158.6,157.9,152.4,138.8,129.3,128.8,113.6$, $111.5,82.3,78.2,59.3,55.4,33.8,28.1,26.0,14.7$; IR (KBr) $v: 3411,3295,2979,2934,2905,2881,1752,1699$, $1672,1613,1510,1462,1372,1303,1288,1262,1249$, 1231, 1203, 1170, 1142, 1107, 1073, 1048, 1035, 951, 848, 785, 774, $746 \mathrm{~cm}^{-1}$; HRMS calcd for $\mathrm{C}_{23} \mathrm{H}_{28} \mathrm{~N}_{2} \mathrm{O}_{7} \mathrm{Na}$ $[\mathrm{M}+\mathrm{Na}]^{+}$467.1794, found 467.1805.

2-氨基-3-甲氧基羰基-5-氧代-4-邻氯苯基-7,8-二氢 化-4H-吡喃并 $[3,2-c]$ 吡啶-6(5H)-羧酸叔丁酯 $(4 \mathbf{p})$ : m.p. 199 201 ${ }^{\circ} \mathrm{C} ;{ }^{1} \mathrm{H}$ NMR (DMSO- $\left.d_{6}, 400 \mathrm{MHz}\right) \delta$ : 1.41 (s, $\left.9 \mathrm{H}, 3 \mathrm{CH}_{3}\right), 2.58 \sim 2.64(\mathrm{~m}, 1 \mathrm{H}, \mathrm{CH}), 2.79 \sim 2.87(\mathrm{~m}, 1 \mathrm{H}$, $\mathrm{CH}), 3.44 \sim 3.51\left(\mathrm{~m}, 4 \mathrm{H}, \mathrm{CH}_{3} \mathrm{O}+\mathrm{CH}\right), 3.95 \sim 4.01(\mathrm{~m}, 1 \mathrm{H}$, $\mathrm{CH}), 4.91 \sim 4.92(\mathrm{~m}, 1 \mathrm{H}, \mathrm{CH}), 7.11 \sim 7.16(\mathrm{~m}, 1 \mathrm{H}, \mathrm{ArH})$, $7.20 \sim 7.28(\mathrm{~m}, 3 \mathrm{H}, \mathrm{ArH}), 7.63\left(\mathrm{~s}, 2 \mathrm{H}, \mathrm{NH}_{2}\right) ;{ }^{13} \mathrm{C} \mathrm{NMR}$ (DMSO- $\left.d_{6}, 100 \mathrm{MHz}\right) \delta: 168.8,163.2,159.5,159.40$, $159.35,152.1,143.1,133.2,132.4,129.9,128.1,127.2$, 109.6, 82.3, 76.5, 50.8, 33.5, 28.1, 26.0; IR (KBr) v: 3440, 3322, 2972, 2936, 2889, 1769, 1700, 1612, 1519, 1269 , $1435,1371,1313,1301,1206,1142,1100,1078,1035$, $952,850,820,784,761,747,705 \mathrm{~cm}^{-1}$; HRMS calcd for $\mathrm{C}_{23} \mathrm{H}_{23} \mathrm{~N}_{2} \mathrm{O}_{6} \mathrm{Cl}[\mathrm{M}+\mathrm{Na}]^{+}$457.1142, found 457.1143.

\section{References}

[1] Almansour, A. I.; Kumar, R. S.; Arumugam, N.; Sriram, D. Eur. J. Med. Chem. 2012, 53, 416.

[2] Girgis, A. S.; Ismail, N. S. M.; Farag, H. Eur. J. Med. Chem. 2011, 46, 2397.

[3] Paronikyan, E. G.; Akopyan, Sh. F.; Noravyan, A. S.; Dzhagatspanyan, I. A.; Nazaryan, I. M.; Akopyan, A. G. Pharm. Chem. J. 2010, 44, 183

[4] Lee, S.; Chae, S. M.; Yi, K. Y.; Kim, N.; Oh, C. H. Bull. Korean Chem. Soc. 2005, 26, 619 .

[5] Van Meir, E. WO 2011133659, 2011 [Chem. Abstr. 2011, 155, 562761].

[6] Finlay, H. J.; Lloyd, J.; Nyman, M.; Conder, M. L.; West, T.; Levesque, P.; Atwal, K. Bioorg. Med. Chem. Lett. 2008, 18, 2714.

[7] Rostamizadeh, S.; Shadjou, N.; Hasanzadeh, M. J. Chin. Chem. Soc. 2012, 59, 866.

[8] Damavandi, S. Synth. React. Inorg., Met.-Org., Nano-Met. Chem. 2012, 42, 1251.

[9] Hafiz, I. S. A.; Ramiz, M. M. M.; Elian, M. A. J. Chem. Sci. 2012 $124,647$.

[10] Goodman, S. N.; Mans, D. M.; Sisko, J.; Yin, H. Org. Lett. 2012, $14,1604$.

[11] (a) Kornienko, A.; Magedov, I. V.; Rogelj, S. US 20090247566, 2009 [Chem. Abstr. 2009, 151, 418130].

(b) Magedov, I. V.; Manpadi, M.; Ogasawara, M. A.; Dhawan, A. S.; Rogelj, S.; Van slambrouck, S.; Steelant, W. F. A.; Evdokimov, 
N. M.; Uglinskii, P. Y.; Elias, E. M.; Knee, E. J.; Tongwa, P.; Antipin, M. Y.; Kornienko, A. J. Med. Chem 2008, 51, 2561.

(c) Magedov, I. V.; Manpadi, M.; Evdokimov, N. M.; Elias, E. M.; Rozhkova, E.; Ogasawara, M. A.; Bettale, J. D.; Przheval'skii, N. M.; Rogelj, S.; Kornienko, A. Bioorg. Med. Chem. Lett. 2007, 17, 3872.

(d) Tokmakov, G. P.; Przheval'skii, N. M.; Rozhkova, E. N.; Nam, N. L. Izv. Timiryazevsk. S-kh. Akad. 2009, 2, 169.

[12] (a) Selvam, N. P.; Babu, T. H.; Perumal, P. T. Tetrahedron 2009, 65,8524 .

(b) Hajbi, Y.; Suzenet, F.; Khouili, M.; Lazar, S.; Guillaumet, G. Synlett 2009, 92.

(c) El-Essawy, F. A.; Khattab, A. F.; Zahran, M. A.; Gomaa, S. H. Synth. Commun. 2007, 37, 3225.

(d) Tchabanenko, K.; Taylor, M. G. O.; Adlington, R. M.; Baldwin, J. E. Tetrahedron Lett. 2006, 47, 39.

(e) Fan, X.; Feng, D.; Qu, Y. Y.; Zhang, X. Y.; Wang, J. J.; Loiseau, P. M.; Andrei, G.; Snoeck, R.; Clercq, E. D. Bioorg. Med. Chem. Lett. 2010, 20, 809.

(f) Stoyanov, E. V.; Ivanov, I. C.; Heber, D. Molecules 2000, 5, 19.

[13] Howarth, J.; James, P.; Dai, J. F. Tetrahedron Lett. 2001, $42,7517$.
[14] Xie, H. B.; Zhang, S. B.; Duan, H. F. Tetrahedron Lett. 2004, 45, 2013.

[15] Adama, C. J.; Earle, J. A.; Roberts, G. Chem. Commun. 1998, 2097.

[16] Mathews, C. J.; Smith, P. J.; Welton, T. Chem. Commun. 2000, 1249.

[17] Xie, X. G.; Chen, B.; Lu, J. P.; Han, J. J.; She, X. G.; Pan, X. F. Tetrahedron Lett. 2004, 45, 6235.

[18] Carmichael, A. J.; Earle, M. J.; Holbrey, J. D.; McCormac, P. B.; Seddon, K. R. Org. Lett. 1999, 1, 997.

[19] (a) Dupont, J.; de Souza, R. F.; Suarez, P. A. Z. Chem. Rev. 2002, 102,3667 .

(b) Martins, M. A. P.; Frizzo, C. P.; Moreira, D. N.; Zanatta, N.; Bonacorso, H. G. Chem. Rev. 2008, 108, 2015.

(c)Welton, T. Chem. Rev. 1999, 99, 2071.

[20] (a) Wang, X. S.; Wu, J. R.; Zhou, J.; Tu, S. J. J. Comb. Chem. 2009, $11,1011$.

(b) Wang, X. S.; Yang, K.; Zhou, J.; Tu, S. J. J. Comb. Chem. 2010, $12,417$.

(c) Wang, S. L.; Yang, K.; Wang, X. S. Chin. J. Org. Chem. 2011, 31, 1235 (in Chinese).

(王树良, 杨科, 王香善, 有机化学, 2011, 31, 1235.) 